\title{
Satisfação global com a vida e determinados domínios entre idosos com amputação de membros inferiores ${ }^{1}$
}

\author{
Maria José D'Elboux Diogo²
}

RESUMO Objetivo. Em idosos com amputação de membros inferiores, atendidos no ambulatório de amputados de um hospital universitário do Município de Campinas, São Paulo, Brasil, identificar relações entre a satisfação global com a vida e a capacidade funcional; avaliar o grau de satisfação com os domínios saúde, capacidade física/dependência, capacidade mental e integração/envolvimento social; e identificar relações entre a satisfação global com a vida e a satisfação com os domínios.

Métodos. Foram avaliados 40 idosos (30 homens e 10 mulheres), com idade média de 74,5 anos (60 a 79 anos), submetidos à amputação acima dos maléolos há no mínimo 1 ano e em acompanhamento para reabilitação entre junho de 1994 e junho de 1999. O instrumento utilizado para a coleta de dados foi composto por: 1) avaliação da capacidade funcional (índice de Barthel); 2) avaliação da satisfação global com a vida (modelo de Cantrill); e 3) avaliação da satisfação com os domínios saúde, capacidade física/dependência, capacidade mental e integração/ envolvimento social, em termos individuais e em comparação com outros idosos.

Resultados. Os participantes apresentaram elevado nível de satisfação global com a vida (média de 7,1; mediana de 7,5; desvio padrão de 2,73). Não houve correlação significativa entre a satisfação global com a vida e a capacidade funcional. Não houve diferença significativa entre o grau de satisfação individual com os diferentes domínios e a percepção desses domínios na comparação com outras pessoas da mesma idade; em ambos os casos, a satisfação observada foi alta. Houve correlação significativa apenas entre a satisfação global com a vida e os domínios saúde (individual e comparada) e capacidade física (comparada).

Conclusões. Os resultados sugerem uma tendência dos idosos com amputação a expressarem uma avaliação positiva sobre seu bem-estar. Esta observação, aliada à escassez de estudos sobre o assunto, justifica a realização de novas pesquisas sobre o bem-estar subjetivo da população idosa e permite um novo olhar sobre a reabilitação dos idosos com amputação de membros inferiores.

Palavras-chave Reabilitação, idoso, amputação, qualidade de vida.

1 Trabalho desenvolvido com base na tese apresentada por ocasião do Concurso de Livre Docência do Departamento de Enfermagem, Faculdade de Ciências Médicas, Universidade de Campinas (UNICAMP). Pesquisa financiada pela Fundação de Amparo à Pesquisa do Estado de São Paulo (FAPESP)

2 UNICAMP, Faculdade de Ciências Médicas, Departamento de Enfermagem. Correspondência e pedidos de separatas devem ser enviados ao seguinte endereço: Cidade Zeferino Vaz, Distrito Barão Geraldo, CEP 13081-970, Campinas, SP, Brasil. E-mail: mariadio@uol.com.br
Nas pesquisas sobre o desenvolvimento do adulto, existe consenso acerca da influência direta que as condições objetivas e subjetivas exercem sobre a qualidade de vida das pessoas. $\mathrm{Na}$ velhice, a qualidade de vida é um evento determinado por múltiplos fatores, os quais nem sempre são fáceis de serem cientificamente avaliados.
Um importante indicador da qualidade de vida, especialmente na velhice, é a capacidade funcional, que pode ser mais preditiva do estado de saúde do idoso do que os próprios diagnósticos médicos $(1,2)$. Entre os fatores objetivos, destacam-se condições de saúde, relações sociais, grau de escolaridade, realização de ativida- 
des e renda, entre outros. Pesquisas mostram que o bem-estar físico objetivo está diretamente relacionado à ausência de doença ou de comprometimento, mesmo que leve, da capacidade funcional e do conforto; portanto, uma boa saúde física seria um forte indicativo de bem-estar psicológico satisfatório $(3,4)$. Quanto às condições subjetivas, dizem respeito ao bem-estar psicológico, ou seja, às experiências pessoais, aos estados internos que podem ser manifestados por meio de sentimentos, às reações afetivas e aos construtos psicológicos como felicidade, satisfação, saúde mental, senso de controle, competência social, estresse e saúde percebida (5).

Os indicadores objetivos nem sempre nos apontam como os indivíduos percebem e experienciam suas vidas. Os indicadores subjetivos, que incluem, por exemplo, a satisfação e a felicidade, definem com maior precisão a experiência de vida em relação às várias condições de vida do indivíduo. São os indicadores mais freqüentemente utilizados nas avaliações de qualidade de vida percebida. Enquanto subjetiva, a avaliação da satisfação com a vida reflete as expressões de cada pessoa quanto a seus próprios critérios de satisfação com a vida como um todo e em domínios específicos, como saúde, trabalho, condições de moradia, relações sociais e outros. Assim, reflete, em parte, o bem-estar subjetivo individual, ou seja, o modo e os motivos que levam as pessoas a viverem suas experiências de vida de maneira positiva. Há três aspectos centrais do bem-estar subjetivo: primeiro, ele reside na experiência do indivíduo; segundo, não compreende somente a ausência de fatores negativos, mas, também, medidas positivas; e, terceiro, inclui, de preferência, uma avaliação global anterior à avaliação de um determinado domínio da vida (6).

A prática assistencial, em nível ambulatorial, com idosos submetidos à amputação, gera várias inquietações acerca das conseqüências da amputação na vida dessas pessoas, entre elas a questão da satisfação com a própria vida. Estariam esses idosos satisfeitos com a sua vida no decorrer do processo de reabilitação? A capacidade funcional seria um elemento determinante na sua avaliação de satisfação com a vida?

Conforme já apontado anteriormente (7), um levantamento bibliográfico realizado como parte do presente trabalho revelou que a maioria dos estudos já publicados tratam dos aspectos funcionais de reabilitação, fatores de impacto como a idade e a co-morbidade. Assim, os objetivos do presente estudo foram: 1) identificar a existência de relações entre a satisfação global com a vida de idosos submetidos à amputação e sua capacidade funcional; 2) avaliar o grau de satisfação desses idosos com os domínios saúde, capacidade física/dependência, capacidade mental e integração/ envolvimento social, em termos individuais e na comparação com outros idosos; e 3) identificar a existência de relações entre a satisfação global desses idosos com a vida e sua satisfação com cada um dos domínios.

\section{MATERIAIS E MÉTODOS}

Participaram deste estudo 40 idosos (30 homens e 10 mulheres), com idade média de 74,5 anos (60 a 79 anos), submetidos à amputação acima dos maléolos há no mínimo 1 ano e em acompanhamento para reabilitação no período de junho de 1994 a junho de 1999. O estudo foi realizado no ambulatório de amputados da Unidade de Órteses e Próteses do Hospital das Clínicas da Universidade Estadual de Campinas (UNICAMP), Campinas, Estado de São Paulo, Brasil. Os idosos foram entrevistados após parecer favorável do Comitê de Ética da instituição e consentimento autorizado conforme legislação vigente no país.

$\mathrm{O}$ instrumento utilizado para a coleta de dados neste estudo foi composto por três partes: 1) avaliação da capacidade funcional segundo o índice de Barthel (8), em que pontuação igual a 100 significa que o indivíduo é totalmente independente, $\geq 60$ indica elevada probabilidade de o indivíduo conviver na comunidade, $\leq 40$ indica importante dependência, e $\leq 20$ indica elevado risco de morte; 2) avaliação global da satisfação com a vida, adaptada do modelo de Cantrill (9), que consiste em uma escala ascendente de 1 a 10, representada esquematicamente por uma escada, na qual o menor valor representa a pior vida e o maior valor, a melhor vida; 3) avaliação da satisfação com os domínios saúde, capacidade física/dependência, capacidade mental e integração/ envolvimento social, em termos individuais e em comparação com outros idosos. Para cada domínio a pontuação variou de 1 (muito pouco satisfeito) a 5 (muitíssimo satisfeito).

$\mathrm{Na}$ análise estatística foi utilizado o coeficiente de Spearman para identificar correlação entre a satisfação global com a vida e o índice de Barthel. Para avaliar a satisfação nos domínios foi utilizado o teste de Wilcoxon para dados relacionados.

\section{RESULTADOS}

Os idosos deste estudo, de um modo geral, apresentaram elevado nível de satisfação global com a vida, com média de 7,1, mediana de 7,5 e desvio padrão de 2,73. A figura 1 mostra que não houve uma relação proporcional entre o índice de Barthel e a satisfação com a vida no momento da entrevista, ou seja, não houve uma tendência comum entre as duas variáveis. $\mathrm{O}$ coeficiente de Spearman também não revelou correlação significativa entre a satisfação global com a vida e o índice de Barthel ( $r=0,26 ; P=0,10)$.

Algumas situações merecem destaque, como por exemplo um idoso que apresentou baixa pontuação no índice de Barthel (40 pontos), porém atribuiu valor máximo a sua satisfação com a vida (10 pontos). Outros dois sujeitos, apesar de apresentarem valores altos no índice de Barthel (90 e 85, respectivamente), avaliaram a satisfação com a vida em níveis baixos (1 e 2 , respectivamente). De um modo geral, observou-se uma concentração de idosos com pontuação alta em Barthel e níveis elevados de satisfação com a vida.

Os resultados referentes ao domínio saúde revelaram que, dos 40 idosos 
FIGURA 1. Diagrama de dispersão da pontuação da satisfação global com a vida e índice de Barthel, pesquisa com idosos amputados, Campinas (SP), Brasil, 2000

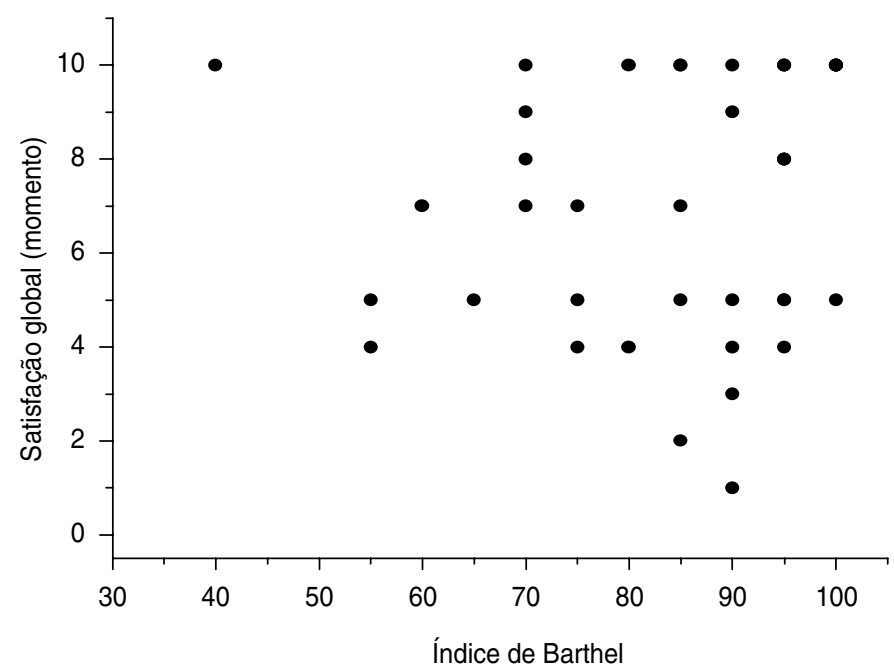

a Embora 40 sujeitos tenham participado do estudo, aparecem somente 30 pontos, em de corrência da sobreposição dos dados.

avaliados, 18 estavam muitíssimo satisfeitos (nível 5) e 13 muito satisfeitos (nível 4) com sua saúde. Somente um sujeito indicou o nível 1, "muito pouco satisfeito", na avaliação individual de sua saúde; este mesmo indivíduo atribuiu o nível 2, "pouco satisfeito" para expressar seu grau de satisfação com a própria saúde em comparação com a saúde de pessoas da mesma idade. Para o grupo como um todo, não houve diferença significativa entre a avaliação individual da saúde e a comparação com a saúde de outras pessoas de mesma idade $(P=0,31)$.

Especificamente em relação ao domínio capacidade física/independência, partiu-se do pressuposto de que os idosos com amputação de membros inferiores tenderiam a atribuir valores menores a sua satisfação quando comparassem o seu grau de capacidade física/independência ao de outras pessoas de mesma idade. No entanto, os resultados contrariaram tal hipótese: 19 idosos disseram estar muitíssimo satisfeitos com a própria capacidade física na avaliação individual, e um número ainda maior - 26 idosos disseram estar muitíssimo satisfeitos quando se compararam com outras pessoas da mesma idade. Nenhum idoso atribuiu valor 1 (muito pouco satisfeito) para esse domínio na comparação com outros idosos; o único idoso que atribuiu o valor mínimo na avaliação individual aferiu valor 5 (muitíssimo satisfeito) quando comparou sua capacidade física com a de outras pessoas da mesma idade. De acordo com o teste de Wilcoxon, não houve diferença significativa $(P=0,11)$ entre o nível de satisfação individual e a comparação com outras pessoas da mesma idade.

Buscando identificar uma relação entre os valores do índice de Barthel (avaliação objetiva) e a satisfação referente ao domínio capacidade física/ independência (avaliação subjetiva), os idosos foram divididos em três grupos, conforme a pontuação no índice de Barthel: B0 (pontuação entre 40 e 60), B1 (pontuação entre 65 e 80) e B2 (pontuação superior a 80). A partir dessa categorização, constatamos que os idosos com amputação de membros inferiores, mesmo aqueles com pontuação relativamente baixa no índice objetivo de Barthel (valores entre 40 e 60), ou seja, que julgavam ter algum comprometimento da sua independência para as atividades da vida diária, ainda se consideravam satisfeitos com a sua capacidade física/independência na avaliação subjetiva. Não houve correlação entre o índice de Barthel e a avaliação subjetiva para esse domínio (Spearman $\mathrm{r}=0,07 ; P=0,64)$.

Quanto ao domínio capacidade mental, 28 entrevistados apresentaram o nível muitíssimo satisfeito tanto para a satisfação pessoal quanto para a comparação de sua capacidade mental com a de outras pessoas da mesma idade. Também nesse domínio não houve diferença significativa entre a satisfação individual e a satisfação comparada $(P=0,51)$.

No domínio integração/envolvimento social a concentração das respostas em nível mais alto foi mais evidente: 34 idosos estavam muitíssimo satisfeitos com o próprio relacionamento social e 32 referiram o mesmo nível quando se compararam com outras pessoas da mesma idade. Nenhum idoso aferiu níveis inferiores a 3, isto é, "pouco satisfeito" e "muito pouco satisfeito", em nenhuma das avaliações. Também nesse domínio não houve diferença significativa entre a avaliação individual e a comparada $(P=0,41)$.

Os resultados da correlação entre satisfação global com a vida e satisfação pessoal e comparada com os domínios investigados, apresentados na tabela 1 , mostram que houve correlação significativa apenas entre satisfação global com a vida e o domínio saúde (individual e comparada) e capacidade física (comparada). Isso significa que quanto maior a satisfação com a vida, maior a satisfação no domínio saúde e capacidade física, e vice-versa.

\section{DISCUSSÃO}

A perda de um membro pode desencadear nos idosos diferentes percepções sobre o seu bem-estar subjetivo, uma vez que as emoções flutuam ao longo da vida segundo a ocorrência de eventos, o estado psicológico num determinado momento e fatores relacionados à personalidade. Desse modo, mesmo que as experiências emocionais intensas sejam importantes, pas- 
TABELA 1. Correlação entre a satisfação global com a vida e a satisfação com determinados domínios entre idosos com membros inferiores amputados, Campinas (SP), Brasil, 2000

\begin{tabular}{lcc}
\hline \multicolumn{1}{c}{ Domínios } & $\begin{array}{c}\text { Correlação com a satisfação } \\
\text { global (Spearman) }\end{array}$ & $P$ \\
\hline Avaliação individual & & 0,00 \\
Saúde & $r=0,44$ & 0,52 \\
Capacidade física/independência & $r=0,30$ & 0,08 \\
Capacidade mental & $r=0,27$ & 0,27 \\
Integração/envolvimento social & $r=0,17$ & 0,00 \\
Avaliação comparada & $r=0,46$ & 0,01 \\
Saúde & $r=0,37$ & 0,06 \\
Capacidade física/independência & $r=0,29$ & 0,46 \\
Capacidade mental & $r=0,11$ & \\
Integração/envolvimento social &
\end{tabular}

${ }^{a}$ Avaliação da satisfação com os domínios a partir da comparação com outras pessoas da mesma idade.

sado o tempo tais experiências raramente parecem diminuir a avaliação subjetiva das pessoas sobre o seu próprio bem-estar (10). Essas colocações podem justificar, em parte, os níveis de satisfação com a vida relativamente elevados atribuídos pelos idosos neste estudo, cuja média foi 7 , numa escala de 1 a 10 , independentemente dos valores do índice de Barthel. Outrossim, talvez o fato de esses idosos serem independentes funcionalmente tenha contribuído para uma avaliação positiva da satisfação global com a vida.

Algumas pesquisas indicam que o bem-estar subjetivo não reside somente no efeito direto de como as pessoas se sentem fisicamente, mas também no que sua saúde permite que elas façam. De fato, no domínio relacionado à saúde obtivemos elevados níveis de satisfação com a vida, com mediana de valor 4 para a avaliação pessoal e 5 para a avaliação comparativa à saúde de outras pessoas da mesma idade. No domínio capacidade física/independência, além das elevadas pontuações no índice de Barthel, os valores obtidos na avaliação pessoal e comparada também foram elevados, indicando que os idosos estavam satisfeitos com sua capacidade funcional.

Os resultados do presente estudo relacionados à satisfação com os diferentes domínios apontaram altos valores. Estes dados vão ao encontro das afirmativas de autores (11) de que o bemestar subjetivo não se refere apenas a ausência de experiências negativas, ou seja, altos valores de bem-estar subjetivo também indicam presença de sentimentos agradáveis e satisfação com a vida em outros domínios. A literatura aponta que existe uma baixa correlação entre as circunstâncias objetivas e o bem-estar subjetivo, talvez porque as pessoas se adaptam rapidamente aos seus recursos e experiências. Outra sugestão é a de que a experiência de bem-estar é influenciada não somente por condições externas da vida, mas também por características de disposição internas, isto é, compleição.

Nos achados de Ryff (12) os idosos consideraram a saúde como o elemento mais importante e a maior causa para infelicidade, associando a manutenção da funcionalidade e a aceitação das alterações, entre outros, às mudanças positivas relacionadas ao envelhecimento e aos significados de bem-estar. Esses achados podem também justificar os resultados do presente estudo, ou seja: na velhice parece haver um incremento da capacidade individual para aceitar mudanças no caso deste estudo, a própria amputação e suas conseqüências. Os idosos com amputação de membros inferiores, ao expressarem o seu grau de satisfação individual e comparado com outras pessoas de mesma idade em relação aos diferentes domínios, são flexíveis e tendem a ajustar sua atenção cognitiva e expectativas às circunstâncias externas, de modo a adquirir vantagens. O modo pelo qual as pessoas percebem seus próprios potenciais em comparação com outras pode determinar como elas sentem suas próprias vidas e condições (5).

Outros autores (11), ao analisarem estudos sobre o bem-estar subjetivo e a velhice, concluem que a satisfação com a vida não declina com o avançar da idade, mesmo sob influência do decréscimo de recursos pessoais. Ademais, pessoas idosas podem ter baixo nível de aspirações e modelos ou padrões ligados à idade e ajustam seus objetivos aos próprios recursos e competências. Uma vez apresentando baixas expectativas, os idosos são mais facilmente satisfeitos. Outrossim, o declínio de recursos objetivos nos períodos mais tardios da vida pode ser "mascarado" por fatores subjetivos nos julgamentos dos idosos sobre a satisfação com a vida. Por exemplo, os mecanismos de comparação social e o medo de envelhecer muitas vezes são melhores preditores do bem-estar das pessoas idosas do que os meios objetivos, como as condições de saúde.

Os padrões de comparação não foram explicitados nos julgamentos que os idosos com amputação de membros inferiores realizaram sobre seu grau de satisfação individual e seu grau de satisfação quando se compararam a outras pessoas em relação aos domínios avaliados. Portanto, esses julgamentos emergiram de critérios pessoais, que variaram de sujeito para sujeito.

Não encontramos, na literatura, estudos sobre satisfação com a vida entre idosos com amputação. Alguns trabalhos tratam dos efeitos da amputação (13), onde as limitações físicas, a depressão, as limitações sociais e a dependência/necessidade de ajuda foram as maiores conseqüências após a perda do membro. Os dados obtidos no presente estudo apontam para a importância de se investir em pesquisas sobre o bem-estar subjetivo da população idosa e poderão, ainda, subsidiar os profissionais, especialmente da área da saúde, em suas práticas assistenciais, visando a valorização da satisfação com a vida e promovendo um novo olhar sobre a reabilitação dos idosos com amputação de membros inferiores. 


\section{REFERÊNCIAS}

1. Neri AL. Qualidade de vida na velhice. Campinas: Papirus; 1993. (Coleção vivaidade).

2. Neri AL. Qualidade de vida na velhice e atendimento domiciliário. Em: Duarte YAO, Diogo MJDE, eds. Atendimento domiciliário: um enfoque gerontológico. São Paulo: Atheneu; 2000. Pp. 33-47.

3. Lawton MP. Environment and other determinants of well-being in older people. Gerontologist 1983;23(4):85-89.

4. Lawton MP. A multidimensional view of quality of life in frail elders. Em: Birren JE, Lubben JE, Rowe JC, Deutchman DE, eds. The concept and measurement for quality of life in the frail elderly. San Diego: Academic Press; 1991. Pp. 3-27.

5. Diener E, Suh E. Measuring quality of life: economic, social, and subjective indicators. Soc Ind Res 1997;40:189-216.
6. Diener E. Assessing subjective well-being: progress and opportunities. Soc Ind Res 1994; 31:103-157.

7. Cutson TM, Bongiorni DR. Rehabilitation of the older lower limb amputee: a brief review. J Am Geriatr Soc 1996;44(11):1388-1393.

8. Mahoney FI, Barthel DW. Functional evaluation: the Barthel Index. Md State Med J 1965; 14:61-65.

9. Cantrill $\mathrm{H}$. The pattern of human concerns New Brunswick, NJ: Rutgers University Press; 1967.

10. Diener E, Larsen R. The experience of emotional well-being. Em: Lewis M, Haviland JM, eds. Handbook of emotions. New York: Guilford; 1993. Pp. 405-415.

11. Diener E, Suh E. Subjective well-being and age: an international analysis. Em: Schaie KW, Lawton MP. Annual review of gerontology and geriatrics. Focus on emotion and adult development. New York: Springer Publishing Company; 1997. Pp. 304-324.

12. Ryff CD. In the eye of the beholder: views of psychological well-being among middle-aged and older adults. Psychol Aging 1989;4(2): 195-210.

13. Thompson DM, Haran D. Living with an amputation: what it means for patients and their helpers. Int J Rehabil Res 1984;7(3):283-292.

Manuscrito recebido em 22 de março de 2002. Aceito em versão revisada em 15 de novembro de 2002
ABSTRACT

Satisfaction with life overall and with specific life domains among elderly persons with a lower limb amputation
Objective. To examine a group of elderly persons who had had a lower limb amputation and who were receiving care at the outpatient clinic of a teaching hospital in Campinas, São Paulo, Brazil, in order to: 1) identify relationships between their perceived overall life satisfaction and their functional capacity; 2) assess their perceived satisfaction with the domains of health, physical functioning/dependence, mental health, and social integration/involvement; and 3) identify relationships between overall life satisfaction and satisfaction with the specific domains.

Methods. An assessment was done of a group of 40 elderly persons (30 men and 10 women) who had had an amputation above the malleolus at least 1 year earlier and who were undergoing rehabilitation between June 1994 and June 1999. Their mean age was 74.5 years (range, 60 to 79 years). A questionnaire was used for data collection to assess: 1) functional capacity (Barthel index); 2) general life satisfaction (Cantrill model); and 3) satisfaction with the domains of health, physical functioning/ dependence, mental health, and social integration/involvement, with the 40 elderly persons assessing themselves both in individual terms and in comparison to other persons of the same age group.

Results. Among the 40 elderly persons, the level of general life satisfaction was high. Measured on a scale of 1 to 10 , the mean was 7.1 and the median 7.5 , with a standard deviation of 2.73. There was no correlation between overall life satisfaction and functional capacity. There was no significant difference between individual perceived satisfaction with the specific domains and the perception of these domains in comparison to other persons of the same age group; in both of these cases, the elderly persons who had had an amputation reported a high degree of satisfaction. A positive correlation was found only between overall life satisfaction and the domains of health (individual and comparative) and of physical functioning (comparative).

Conclusions. These results suggest that this group of elderly persons who had had an amputation tended to assess their situation positively. This finding provides new insights into the rehabilitation of elderly persons with lower limb amputations. Taken together with the dearth of studies concerning the subject, this finding also indicates the need for additional research focusing on the subjective well-being of the elderly. 